\title{
Behavioural disturbance in people with epilepsy and a mental handicap
}

\author{
Paul McQuall, Registrar in Psychiatry, Academic Sub-Department of Psychological \\ Medicine in North Wales, North Wales Hospital, Denbigh, Clwyd LL16 5SS
}

People with a mental handicap may have a variety of behavioural symptoms, and according to DSMIII-R those commonly seen include passivity, low self-esteem, low frustration tolerance, aggressiveness, poor impulse control, and stereotyped selfstimulating and self-injurious behaviour. There is, however, no diagnostic subclassification of these in DSM-III-R.

People suffering from epilepsy are also known to show various behavioural disorders, both peri-ictally and inter-ictally.

The occurrence of epilepsy in people with a mental handicap has not consistently been shown to be associated with more behavioural disturbance than in those people with a mental handicap alone (Lund, 1985; Espie et al, 1989), but in general the findings suggest that people with more severe epilepsy have more behavioural disturbance.

\section{The study \\ Objectives}

The main objective of this study was to assess the relationship between the degree of behavioural disturbance and the severity of epilepsy in a group of in-patients in Bryn-y-Neuadd Hospital in North Wales, which is a hospital for adults with a mental handicap.

Previous studies in people with a mental handicap and epilepsy have shown that excessive amounts of anticonvulsant medication may contribute to behavioural disturbance (Fischbacher, 1982) and this relationship was also considered in this study. The effects of other variables which have been shown to be associated with behavioural disturbances were also considered, including age, sex and degree of ambulancy (Duker et al, 1986).

\section{Inclusion criteria}

It was decided to include patients with generalised clonic-tonic type seizures who had at least one seizure in the last year.

\section{Data collected}

The study was retrospective and data were collected about the patients on demographic features (age, sex), epilepsy, medication, and behavioural disturbance.
The types of epileptic seizure as well as the number of seizures over the last year were obtained from medical and nursing records, which included a seizure chart.

Data were obtained about anticonvulsant medication over the last year, and about serum anticonvulsant levels. The use of other medication was also recorded.

\section{Behavioural disturbance}

Data on behavioural disturbance were collected from a questionnaire which was completed by interviewing nursing staff involved with the care of the patients, about observed and recorded behavioural problems over the last year. An attempt was made to distinguish peri-ictal behaviour problems from inter-ictal behaviour problems. Eight aspects of behavioural disturbance were rated including aggressive behaviour, emotional (or psychological) disturbance, antisocial conduct, withdrawn behaviour, idiosyncratic mannerisms, self injury, hyperactivity, and abnormal sexual behaviour. Each aspect of behavioural disturbance was rated depending on the frequency of the disturbance either zero (never), one (occasional) or two (frequently). A total score was used as a measure of overall behavioural disturbance (maximum 16).

\section{Findings}

\section{Patient characteristics}

Out of a total hospital population of 193 at the time of the study, there were 57 patients with epilepsy, although 11 of these were excluded from the study because they had no seizures recorded in the last year, leaving a total of 46 .

Of the 46 subjects, 27 were male and 19 female, and the mean age was 38.3 (range 18 to 81 years). The male subjects were older with a mean age of 41.1 compared with the female mean age of 34.3. All the patients were documented as having severe mental handicap. Three were recorded as having a mental disorder, one with schizophrenia, one with depressive disorder, and one with early dementia. Nineteen out of $\mathbf{4 6}$ were non-ambulant, seven were partially ambulant, leaving 20 who were ambulant. 


\section{Seizure characteristics}

All 46 patients were documented as having clonictonic type seizures, and 10 of these patients also had other types of seizure (complex partial seizures and generalised absence seizures). The range of seizure frequency over a year was from 1 to 137 for total seizures and 1 to 129 for grand mal seizures only. The mean seizure frequency was 28.9 (s.d. 34.2), being higher in the male subjects 31.6 (s.d. 36.7 ) than the female subjects 25.3 (s.d. 30.9).

\section{Medication}

All 46 subjects were prescribed anticonvulsant medication, with a range from one to three drugs prescribed, with 17 people on monotherapy, 23 people on two drugs and six people on three drugs. The mean number of drugs prescribed was 1.8 (s.d. 0.7). Male subjects were prescribed more, with a mean of 1.9 compared with a female mean of 1.5 . The most commonly prescribed drug was sodium valproate which was being taken by 33 of the 46 patients, followed by carbamazepine taken by 27 , phenytoin taken by 12 , clobazam taken by seven and primidone taken by one patient.

In general, those subjects with more seizures were prescribed more anticonvulsants (Spearman coefficient $=0.41, P=0.01$ ). Of a total of 71 serum anticonvulsant levels recently measured, the majority were within the recommended therapeutic range (44) or below (23) with only four above the recommended upper limit (three patients taking sodium valproate, one taking carbamazepine), and these showed no obvious adverse effects.

Fifteen of the $\mathbf{4 6}$ people were prescribed phenothiazine medication for behavioural disturbance; one of these had a schizophrenic disorder. Four people were on thyroxine replacement.

\section{Behavioural disturbance}

The results of the behavioural disturbance assessments showed a range of total disturbances scored from 0 to 15 with a mean of 6.2 (s.d. 3.8). None of the scores were thought to reflect peri-ictal activity. Men showed more disturbance overall as a group, with a mean of 6.6 compared with 5.1 for women, although this difference did not reach statistical significance.

\section{Behavioural disturbance and seizure frequency}

There was little correlation between the frequency of epileptic seizures and the degree of total behavioural disturbance (Spearman coefficient $=-0.20$ ). Similarly there were no significant relationships between seizure frequency and the eight individual types of behavioural disturbance.

\section{Behavioural disturbance and anticonvulsant medication}

Total behavioural disturbance was not significantly correlated with the numbers of anticonvulsant drugs taken (Spearman coefficient $=-0.28$ ), and for individual anticonvulsant drugs there were little in the way of significant relationships with behavioural disturbance.

\section{Behavioural disturbance and other factors}

There were no significant correlations between the amount of behavioural disturbance and the age (Spearman coefficient $=-0.01$ ), sex (Spearman coefficient $=-0.17$ ), or degree of ambulancy (Spearman coefficient $=-0.30$ ) in the subjects.

\section{Comment}

\section{Limitations of study}

Ideally such a study should be a longitudinal and prospective one. The limitations of this study include the fairly small size of the sample, the questionable validity of the behavioural assessment questionnaire and the possibility of seizures not being recorded accurately on a 24 hour basis. The effects of certain other variables thought to be related to behavioural disturbance, particularly environmental characteristics such as the staff-patient ratio, were not taken into account.

\section{Findings}

These suggest that the degree of behavioural disturbance in this group of 46 institutionalised people with epilepsy and a mental handicap does not vary with the severity of epilepsy. This finding differs from the few previous similar studies. For instance, a study on 15 in-patients found that those subjects with more seizures had more overall behavioural disturbance (Espie et al, 1989).

The finding that the number of anticonvulsants taken does not seem to effect the degree of behavioural disturbance may reflect the trend to minimise polypharmacy as well as to use safer anticonvulsants which are prescribed within the therapeutic range.

\section{Implications of the findings}

Perhaps it is the case for the severe epileptics in this study that the extra seizures are in some way protective against interictal behavioural disturbance, analogous to the protective effect of seizures in the schizophrenia-like disorders associated with epilepsy. This may offer some comfort for doctors involved in the care of people with such severe epilepsy. 


\section{Acknowledgements}

I would like to acknowledge the kind help of Dr L. Vati, Bryn-y-Neuadd Hospital, and Professor Greg Wilkinson, London Hospital Medical College.

\section{References}

Duker, P., Van Druenen, C., Jol, K. \& Oud, H. (1986) Determinants of maladaptive behaviour of institutionalized mentally retarded individuals. American Journal of Mental Deficiency, 91, 51-56.
Espie, C., Pashley, A., Bonham, K. et al (1989) The mentally handicapped person with epilepsy: a comparative study investigating psychosocial functioning. Journal of Mental Deficiency Research, 33, 123-135.

FischBACHER, E. (1982) Effect of reduction of anticonvulsants on wellbeing. British Medical Journal, 285, 423-424.

LUND, J. (1985) Epilepsy and psychiatric disorder in the mentally retarded adult. Acta Psychiatrica Scandinavica, 72, 557-562.

\title{
Will the information recorded in psychiatric notes change when patients have the right to read them?
}

\author{
RuPERT MCShane, Senior House Officer; DeRmot Rowe, Senior House Officer; and \\ DAVID JULIER, Consultant Psychiatrist, Littlemore Hospital Oxford OX4 4XN
}

Psychiatric patients have had the right to see their notes from 1 November 1991. The 'Access to Health Records Act (1990)' makes provision for certain parts of the record to be exempt, including information which may cause serious mental or physical harm to the patient or anyone else. In addition, patients should not have access to information given by third party informants unless appropriate consent has been obtained. The legislation only applies to records made after 1 November 1991. It does not cover informal arrangements where written application for access is not made.

Several studies have suggested that access to notes may have therapeutic potential for the patient (e.g. Parrott et al, 1988). Patients are more likely to ask to see their notes if they are informed of their right to do so. Nevertheless, worries remain that health care workers and informants will become less than frank in the information which they record or volunteer.

\section{The study and findings}

A postal survey of all GPs, hospital psychiatric doctors and psychiatric nurses in the Oxford District was conducted between 1 June and 15 July 1991.

Questionnaires were sent to 272 GPs, 49 consultant and junior hospital psychiatrists, and 83 qualified RMNs. After a follow-up message to nonresponders the response rates were $83 \%$ from GPs, $90 \%$ from hospital doctors, and $77 \%$ from nurses.

The questionnaire investigated three issues.

(a) If psychiatric patients were routinely informed of their right to see their hospital notes, how inhibited would you have felt about recording an opinion about the personality of the last (in)patient who was referred to/by you?

(b) How important do you think it is that you are told if a psychiatric in-patient is going to see 